\title{
Metal chaperones prevent zinc-mediated cognitive decline
}

\author{
Paul A. Adlard ${ }^{\mathrm{a}, *}$, Jacqui Parncutt ${ }^{\mathrm{d}}$, Varsha Lal ${ }^{\mathrm{d}}$, Simon James ${ }^{\mathrm{b}}$, Dominic Hare ${ }^{\mathrm{c}}$, Philip Doble ${ }^{\mathrm{c}}$, \\ David I. Finkelstein ${ }^{\mathrm{a}}$, Ashley I. Bush ${ }^{\text {a }}$ \\ a The Florey Institute for Neuroscience and Mental Health and The University of Melbourne, Parkville, Victoria 3052, Australia \\ ${ }^{\mathrm{b}}$ Australian Synchrotron and the CSIRO Preventative Health Flagship, Clayton, Victoria 3168, Australia \\ c Elemental Bio-imaging Centre, University of Technology Sydney, Broadway, New South Wales 2007, Australia \\ d The Mental Health Research Institute, Parkville, Victoria 3052, Australia
}

\section{A R T I C L E I N F O}

\section{Article history:}

Received 12 September 2014

Revised 24 November 2014

Accepted 11 December 2014

Available online xxxx

\section{Keywords:}

Zinc

ZnT3

Clioquinol

Animal model

Cognition

\begin{abstract}
A B S T R A C T
Zinc transporter-3 (ZnT3) protein is responsible for loading zinc into presynaptic vesicles and consequently controls the availability of zinc at the glutamatergic synapse. ZnT3 has been shown to decline with age and in Alzheimer's disease $(A D)$ and is crucially involved in learning and memory. In this study, we utilised whole animal behavioural analyses in the ZnT3 KO mouse line, together with electrophysiological analysis of long-term potentiation in brain slices from ZnT3 KO mice, to show that metal chaperones (clioquinol, $30 \mathrm{mg} / \mathrm{kg} / \mathrm{day}$ for 6 weeks) can prevent the age-dependent cognitive phenotype that characterises these animals. This likely occurs as a result of a homeostatic restoration of synaptic protein expression, as clioquinol significantly restored levels of various pre- and postsynaptic proteins that are critical for normal cognition, including PSD-95; AMPAR and NMDAR2b. We hypothesised that this clioquinol-mediated restoration of synaptic health resulted from a selective increase in synaptic zinc content within the hippocampus. While we demonstrated a small regional increase in hippocampal zinc content using synchrotron x-ray fluorescence microscopy, further sub-region analyses are required to determine whether this effect is seen in other regions of the hippocampal formation that are more closely linked to the synaptic plasticity effects observed in this study. These data support our recent report on the use of a different metal chaperone (PBT2) to prevent normal age-related cognitive decline and demonstrate that metal chaperones are efficacious in preventing the zinc-mediated cognitive decline that characterises ageing and disease.
\end{abstract}

(C) 2014 Elsevier Inc. All rights reserved.

\section{Introduction}

The underlying mechanisms of cognitive loss in aging and Alzheimer's disease (AD) have largely remained uncertain. Recent evidence implicates presynaptic $\mathrm{Zn}^{2+}$, released with glutamate and loaded into vesicles by Zinc Transporter-3 (ZnT3), in both the formation of the hallmark $\beta$-amyloid accumulation of AD (Lee et al., 2002) and in normal cognitive function (Adlard et al., 2010). ZnT3 allows $\mathrm{Zn}^{2+}$ to achieve high micromolar concentrations during activity within the glutamatergic synapses governing memory and learning (Frederickson et al., 2006). This $\mathrm{Zn}^{2+}$ may influence the neurochemistry of cognition by acting as a neuronal messenger and a modulator of synaptic transmission and plasticity (Frederickson et al., 2005) through interactions with targets including ZnR (GPR39) (Besser et al., 2009), TrkB (Huang et al., 2008), glutamate receptors (Paoletti et al., 2009) and p75(NTR) (Lee et al., 2008). Indeed, the targeted ablation of ZnT3 in wild-type mice

* Corresponding author at: The Florey Institute of Neuroscience and Mental Health, 30 Royal Parade, Parkville, Victoria 3052, Australia.

E-mail address: paul.adlard@florey.edu.au (P.A. Adlard).

Available online on ScienceDirect (www.sciencedirect.com). results in an impairment in cognitive function that is paralleled by deficits in key proteins involved in synaptic plasticity pathways (Adlard et al., 2010). This led us to hypothesise that ZnT3 KO mice are a phenocopy of the synaptic and memory deficits of AD and potentially also of normal ageing. In this study, we have addressed the hypothesis that the use of metal chaperones will restore metal ion homeostasis within the hippocampus of ZnT3 KO mice to result in a concomitant normalisation of key biochemical pathways involved in cognition and subsequent amelioration of the cognitive loss that characterises this model.

\section{Materials and methods}

\section{Animal tissues}

ZnT3 KO and WT animals were housed in the Animal Facility at the Mental Health Research Institute. All animal experimentation was approved by the Howard Florey Animal Ethics Committee and conformed to Code of Practice established by the National Health and Medical Research Council of Australia for the Care and Use of Animals for Scientific Purposes (7th Edition, 2004). 
Animals (WT and KO; $n=12-18$ /group; equal mix of males and females) were randomly assigned to receive either a sham diet (no supplement), or a diet supplemented with clioquinol (Specialty Feeds, Western Australia). The inclusion rate of drug in the food was $0.25 \mathrm{~g} / \mathrm{kg}$ food, which equates to a daily dose rate of approximately $30 \mathrm{mg} / \mathrm{kg}$ animal weight, based upon on average food intake for rodents, as reported in the ANZCCART Fact Sheet. Animals received these modified diets for 6 weeks prior to culling. Animals were killed at three and 6 months of age. Prior to culling, animals were tested for spatial memory in the Morris water maze and Y-maze.

On completion of behavioural studies, animals were anaesthetised (pentobarbital, IP injection) and transcardially perfused with ice-cold PBS. Brains were removed and hemisected; right hemispheres were further microdissected and frozen on dry ice while left hemispheres were snap-frozen in ice-cold isopentane and prepared for synchrotron analysis.

Separate cohorts of mice were utilised for electrophysiology experiments.

\section{Behavioural studies}

We utilised the Morris water maze as previously reported (Adlard et al., 2008), in addition to the Y-maze as previously reported (Lei et al., 2012) to assess spatial learning and memory. There were no significant differences in swim speeds between groups in the water maze, and we have similarly previously reported that there are no motor differences between the WT and the KO mice. Furthermore, we have also previously reported that this class of compound does not alter the motor activity of animals that do not have a gross motor deficit.

\section{Metal analyses}

Lyophilised hippocampal homogenates ( $n=12-18 /$ group) were analysed by inductively coupled plasma mass spectrometry (ICPMS), as previously reported (Adlard et al., 2010). Briefly, samples were digested in concentrated nitric acid (Aristar, $\mathrm{BDH}$; overnight, room temperature and then $90{ }^{\circ} \mathrm{C}$ for twenty minutes) and then diluted to $1 \%$ and measurements made using a Varian UltraMass inductively coupled plasma mass spectrometer under conditions suitable for routine multi-element analysis. The instrument was calibrated using blank, 10, 50 and $100 \mathrm{ppb}$ of a certified multi-element ICPMS standard solution (ICP-MS-CAl2-1; AccuStandard) for $\mathrm{Mn}, \mathrm{Fe}, \mathrm{Cu}$, and $\mathrm{Zn}$ in $1 \%$ nitric acid. Results were normalised to tissue wet weight before analysis.

For synchrotron analyses, multiple slices (40- $\mu$ m-thick) were prepared from each of the frozen mouse brains using a cryostat before

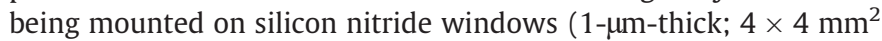
window within a $10 \times 10 \mathrm{~mm}^{2}$ silicon frame). Tissue sections were mounted in order to frame the hippocampus, in particular the zinc rich dentate gyrus and some of the surrounding structures including the major lamellar regions. Sectioned brains were affixed onto the silicon nitride windows by gentle drying under a stream of air. Trace metal distributions in mouse brain sections were mapped using a monochromatic $12.73 \mathrm{keV}$ X-ray beam focused to a spot size of $\sim 2 \mu \mathrm{m}$ in diameter using the Kirkpatrick-Baez mirror microprobe installed at the XFM beamline at the Australian Synchrotron (AS). The sample was raster scanned through the focus of the incident $\mathrm{x}$-ray beam and the resulting elemental fluorescence was recorded using the annular 384-channel monolithic silicon diode detector array (Maia). The detector was oriented in backscatter geometry and subtended an active solid angle of $\sim 1.3 \mathrm{sr}$ and employs a low-latency continuous fly scanning approach, leading to zero readout overhead. The system achieves very short effective dwell times for typically dilute biological specimens $(\sim 10 \mathrm{~ms})$, allowing elemental distributions to be mapped over anatomically relevant structures without sacrificing resolution or sensitivity. The pixel size of $1.25 \times 1.25 \mu \mathrm{m}^{2}$ used in these studies was well matched to the beam spot size, and the resulting full-spectral images ranged up to 1 Mpixel in size. The deconvolution of the Maia data was performed using the DA method and GeoPIXE software suite that incorporates a linear transformation matrix to perform spectral deconvolution to the spectrum recorded at each pixel. Spectral deconvolution was calibrated using a thin Pt foil, and corrections were made for self-absorption in the sample, absorption in air, and the efficiency response of the detector, including absorption in a Mo mask used to reject X-rays that may otherwise produce charge sharing between detectors. The detected X-ray signals in each pixel are related to calculated model fluorescence X-ray yields for an assumed specimen composition and thickness. Absorption effects at the lowest atomic number element that can be accessed using the Maia detector (potassium Ka radiation) are negligible for these tissue sections, assuming typical tissue composition and an average density of $1.04 \mathrm{~g} / \mathrm{cm}^{3}$, typical of brain matter.

\section{Electrophysiology}

For long-term potentiation studies, we utilised WT and ZnT3 KO mice at 3 and 6 months of age. Brain slices ( $n=4-7$ per group) were cut at $400 \mu \mathrm{m}$ thickness using a Vibratome slicer and slices equilibrated at $28^{\circ} \mathrm{C}$ for 2 hours prior to experimentation. For the LTP rescue studies, we supplemented slices with either zinc $(4 \mu \mathrm{m})$, Clioquinol (CQ $4 \mu \mathrm{m})$ or a combination of both for 30 min prior to recording. For electrophysiological recordings, the stimulating electrode was placed on the Schaffer collaterals and the recording electrode in the dendrites of the CA1 pyramidal neurons. The postsynaptic activity of the neurons was recorded by initially identifying the population spike, and then baseline was recorded for 15 minutes at $50 \%$ of maximum activity. Following tetanic stimulation $(100 \mathrm{~Hz}, 1 \mathrm{~s})$, extracellular evoked synaptic activity was recorded for one hour and LTP quantitated. The change in field excitatory postsynaptic potentials relative to baseline averaged from data taken 55 to 60 min posttetanus is shown.

\section{Biochemical analyses}

Western blot was used for quantification of protein levels, as previously reported (Adlard et al., 2010). Briefly, hippocampal homogenates ( $n=8$ /group) were prepared and run on NuPage Novex 4-12\% Bis-Tris gels (Invitrogen; $130 \mathrm{~V}, 90 \mathrm{~min}$ ), transferred to nitrocellulose using the iBlot Gel Transfer Device (Invitrogen; program 3), and the resulting membrane prepared for blotting (heating, blocking). Primary antibodies were incubated on the membranes overnight $\left(4^{\circ} \mathrm{C}\right)$, followed by rinsing and incubation in secondary antibody ( $1 \mathrm{hr}$, room temperature), further rinsing and subsequent development with ECL reagent and imaging (Fujifilm LAS-3000).

\section{Statistical analysis}

Data were analysed using JMP version 5.0.1a (SAS). For statistical comparisons of water maze data, repeated measures ANOVA were conducted, followed by independent one-way ANOVAs for each inter-day comparison between groups (as was also done for other two sample comparisons in this study).

\section{Results}

\section{Clioquinol restores cognition in ZnT3 KO mice}

We assessed the effect of clioquinol (CQ) $(\sim 30 \mathrm{mg} / \mathrm{kg}$, incorporated into regular rodent chow, Specialty Feeds, 6 weeks) in a cohort of ZnT3 KO mice $(n=15)$, as compared to untreated ZnT3 KO mice $(n=16)$. Previously we have demonstrated that 6-month-old ZnT3 KO mice exhibit a marked impairment in learning (overall ANOVA, $p<$ $0.0001)$ and memory $(p<0.0001)$ in the Morris water maze, as compared to age-matched wild-type controls (Adlard et al., 2010). Six 
weeks of CQ treatment fully restored cognitive function (both the learning and the memory components of the Morris water maze) in these animals, to levels equivalent to WT mice (Figs. 1a, b). We further assessed

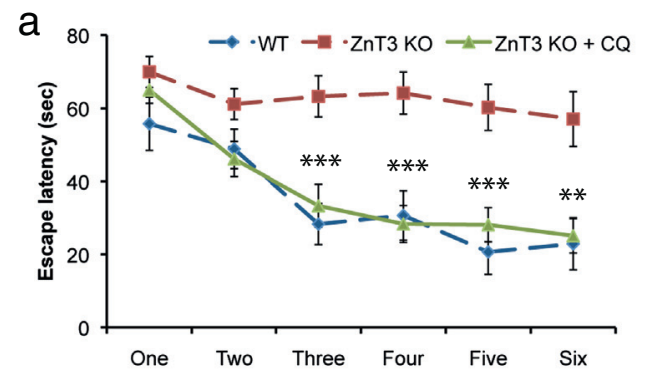

C

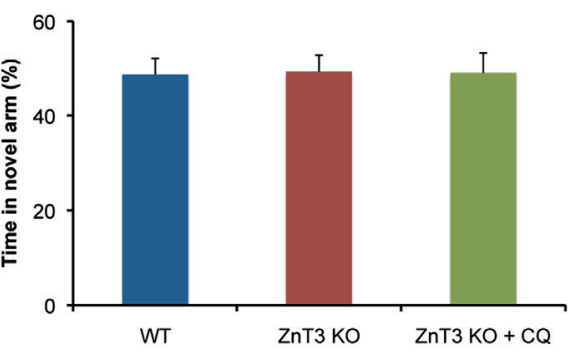

e

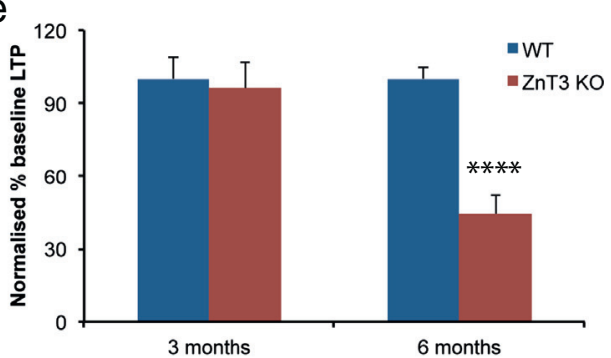

g

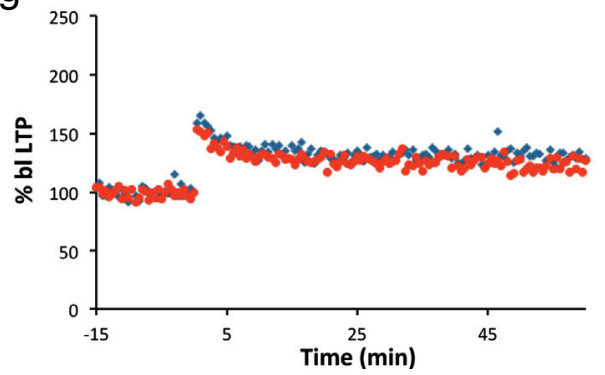

three and 6-month-old ZnT3 KO mice in the Y-maze. Younger ZnT3 KO animals performed identically to age-matched WTs (Fig. 1c), consistent with the Morris water maze data we have previously published. In

\section{b}

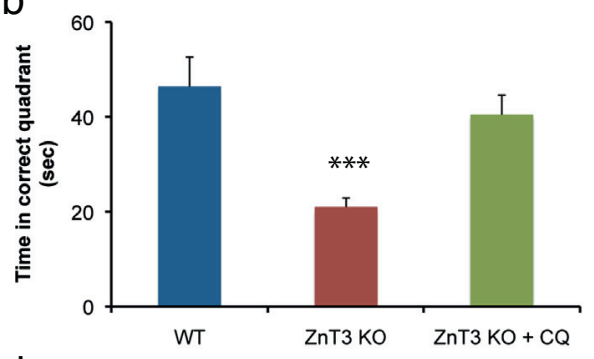

d

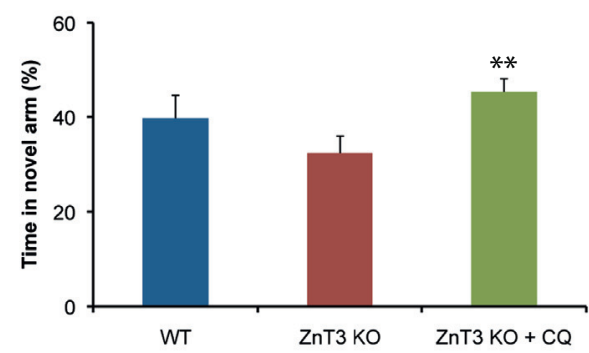

f

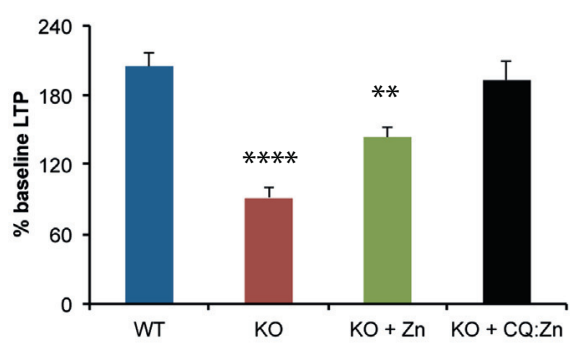

$\mathrm{h}$

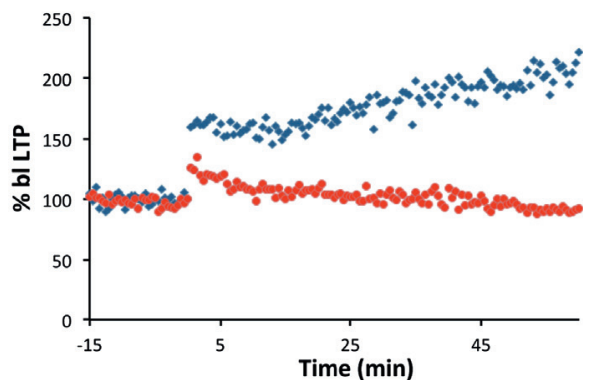

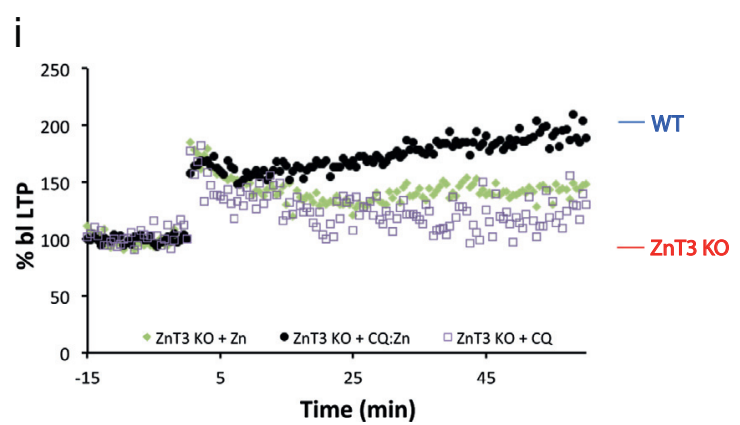

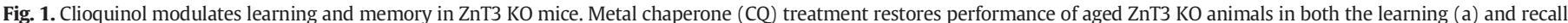

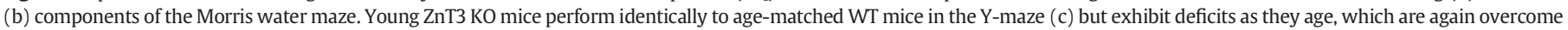

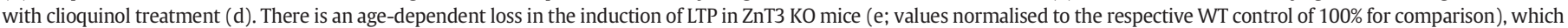

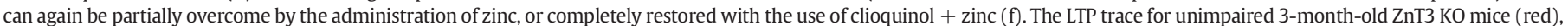

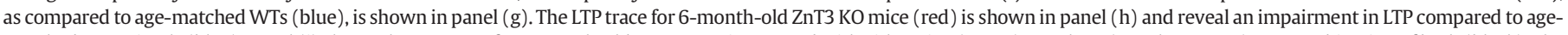

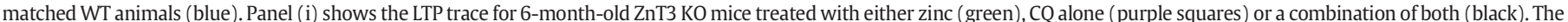

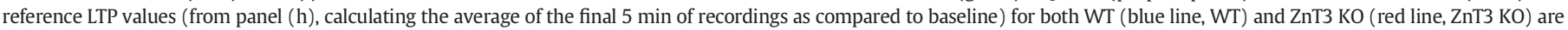

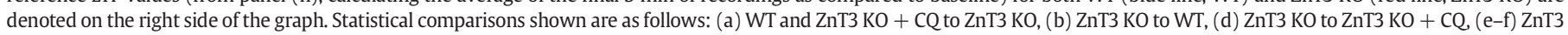
KO to WT; in all cases, ${ }^{* *} p<0.01,{ }^{* * *} p<0.001,{ }^{* * * *} p<0.0001$. KO $=$ ZnT3 KO 
contrast, aged ZnT3 $\mathrm{KO}$ animals exhibited deficits in this task (overall ANOVA, $p=0.01$; WT to ZnT3KO comparison, $p=0.04$ ), again consistent with our earlier work, that were overcome with CQ treatment (Fig. 1d) (ZnT3 KO to ZnT3 KO + CQ, $p=0.007$ ). To further explore the effect of the ablation of ZnT3 on cognition, we examined baseline long-term potentiation (LTP) in young and aged mice to determine whether gross behavioural abnormalities also translated to molecular surrogates of learning and memory such as LTP. Consistent with the behavioural studies, young ZnT3 KO animals were indistinguishable from their wild-type counterparts (Figs. 1e, g), and the aged animals showed a pronounced impairment in their induction of LTP (Figs. 1e, h) $(-56 \%, p<0.0001)$. This deficit was partially prevented by the addition of $\mathrm{Zn}(+26 \%, p<0.01)$ and completely abrogated by the addition of CQ:zinc ( $+50 \% . p<0.0001$ ) (Figs. 1f, i). There remained a significant difference between WT and ZnT3 KO + zinc $(p<0.01)$, but no difference between WT and ZnT3 KO + CQ:zinc. There was also a significant difference between $\mathrm{ZnT} 3 \mathrm{KO}+$ zinc and ZnT3 KO + CQ:zinc $(p<0.01)$. These data support the notion that the loss of synaptic zinc results in a cognitive impairment that can be restored through the use of metal chaperones.

\section{Clioquinol restores levels of key proteins in ZnT3 KO mice}

We have previously reported that aged ZnT3 KO mice have deficits in key proteins related to synaptic plasticity, as also shown in Table 1 (Adlard et al., 2010). We report here that 6 weeks of CQ treatment, which lacks any nootropic effects in normal mice, fully restored cognitive function in aged ZnT3 KO mice concomitant with a significant restoration of key neurochemical deficits (Table 1). Proteins that were changed with $\mathrm{CQ}$ treatment include the following: presynapticSNAP-25 $(+45 \%, p<0.001)$, synaptophysin $(+68 \%, p<0.05)$; postsynaptic-spinophilin $(+36 \%, p<0.05)$, PSD-95 $(+19 \%, p<0.05)$; cell support-pro-BDNF $(+15 \%, p<0.05)$, DCX $(+32 \%, p<0.001)$; glutamate receptors-AMPAR $(+26 \%, p<0.01)$, NMDAR2a $(+43 \%, p<0.05)$ and NMDAR2b $(+94 \%, p<0.001)$. With the exception of NMDAR2b, in all cases where there were protein deficits in the aged ZnT3 KO (compared to age-matched WT), CQ treatment significantly elevated these markers to be more closely aligned with that seen in age-matched WTs. In the case of NMDAR2b, however, there was a significant elevation/"overshoot" above baseline in hippocampal protein levels, which is not inconsistent with the observed improvement in cognition.
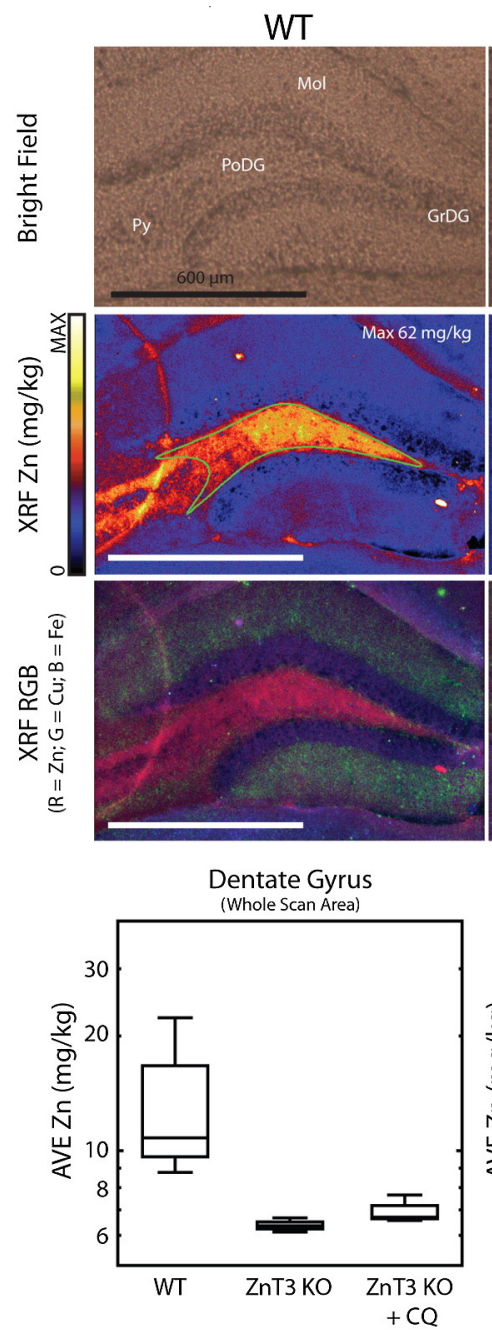
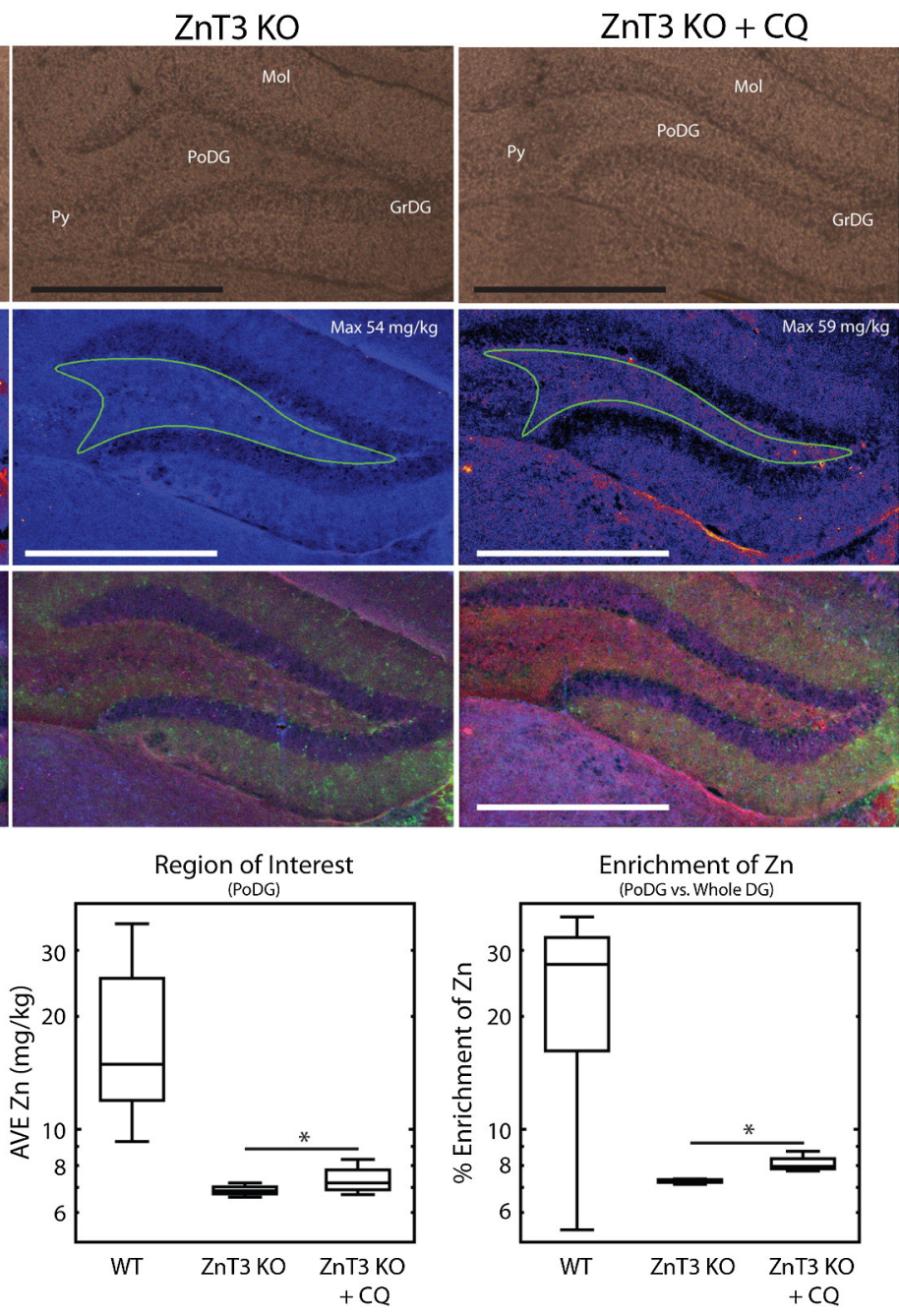
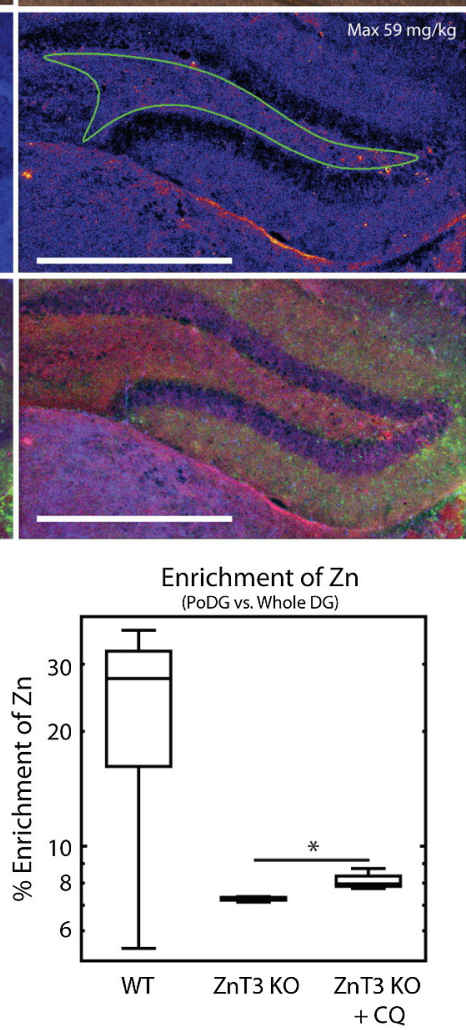

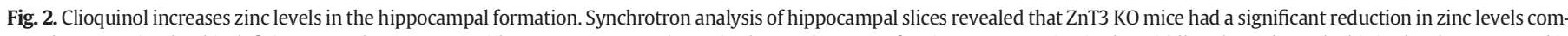

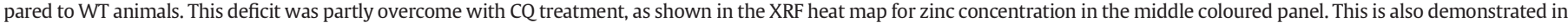

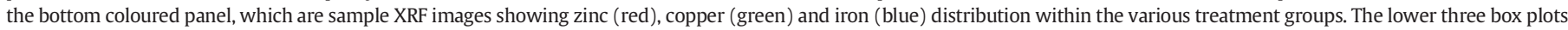

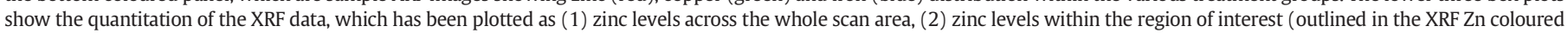
panels) and (3) the enrichment of zinc (PoDG vs the entire dentate gyrus). These data reveal a significant increase in zinc within the dentate gyrus. ${ }^{*}<<0.05$. 
Table 1

Clioquinol restores biochemical parameters of learning and memory in ZnT3 KO mice Values shown are mean \pm SEM Western blot densities normalised to $100 \%$ of agematched WT means from hippocampal homogenates prepared from 6-month-old mice.

\begin{tabular}{|c|c|c|}
\hline & ZnT3 KO & ZnT3 KO + CQ \\
\hline \multicolumn{3}{|l|}{ Presynaptic } \\
\hline SNAP-25 & $53.7 \pm 3.3^{* *}$ & $99 \pm 8.8^{\# \# \#}$ \\
\hline Synaptotagmin I & $123 \pm 12.5$ & $128 \pm 12.9$ \\
\hline Synaptophysin & $142.4 \pm 18^{*}$ & $210 \pm 14.5^{* * * \#}$ \\
\hline \multicolumn{3}{|l|}{ Postsynaptic } \\
\hline Spinophilin & $114 \pm 7.9$ & $150 \pm 14.1^{* * \#}$ \\
\hline PSD-95 & $62.7 \pm 5.7^{* *}$ & $81 \pm 3.8^{* \#}$ \\
\hline \multicolumn{3}{|l|}{ Cell support } \\
\hline TrkB & $78 \pm 4.8^{* *}$ & $87 \pm 8.4$ \\
\hline pro-BDNF & $70.8 \pm 2.8^{*}$ & $85 \pm 4^{\#}$ \\
\hline BDNF & $86.7 \pm 4.8$ & $96 \pm 6.7$ \\
\hline DCX & $69 \pm 4.5^{* * *}$ & $101 \pm 7.5^{\# \# \#}$ \\
\hline \multicolumn{3}{|l|}{ Glutamate receptors } \\
\hline AMPAR & $66 \pm 6.5^{* *}$ & $92 \pm 6.9^{\# \#}$ \\
\hline NMDAR1 & $83.2 \pm 13$ & $98.8 \pm 13$ \\
\hline NMDAR2a & $36.3 \pm 6^{* * *}$ & $79 \pm 14.7^{\#}$ \\
\hline NMDAR2b & $50.9 \pm 9.8^{*}$ & $144 \pm 15.3^{\# \# \# ~}$ \\
\hline
\end{tabular}

*Significantly different to age-matched WT.

\#For CQ-treated animals, significantly different to age-matched ZnT3 KO.

\section{Clioquinol elevates hippocampal zinc levels in ZnT3 KO mice}

We have recently provided evidence for our proposed mechanism of action of metal chaperones, which involves the maintenance of metal ion homeostasis and subsequent downstream effects on metaldependent proteins and signalling pathways (Adlard et al., 2008, 2014; Crouch et al., 2011). To assess the effect of CQ on metal levels in this study, we examined hippocampal zinc levels using both ICPMS and synchrotron X-Ray Fluorescence Microscopy. The age-related reduction in bulk hippocampal zinc levels in the ZnT3 KO mice $(-33 \%)$ was partially restored $(+8 \%)$ by CQ treatment and was more conspicuous when normalised against hippocampal copper levels (ZnT3KO, $2.55 \pm 0.09$; ZnT3 KO + CQ $3.04 \pm 0.2,+19 \%, p=0.03$ ). Synchrotron analysis was then performed in a sub-region of the hippocampus (see Fig. 2). Within the limited area of tissue able to be scanned for the study (the dentate gyrus), we observed a small but significant enrichment of $\mathrm{Zn}$ in the CQ treatment group $(+11 \%, p<0.05)$. While larger studies are required to assess more global changes, it is apparent from both this and our previous study (Adlard et al., 2014) that acute metal chaperone treatment results in a redistribution of $\mathrm{Zn}$ within the metal-deficient hippocampus.

\section{Discussion and conclusion}

We discovered that ZnT3 KO mice recapitulate key synaptic and memory deficits of aging and Alzheimer's disease. These findings support a critical role for zinc signalling in the maintenance of normal cognition. In addition, it prompted the hypothesis that therapeutic strategies that target zinc ion dyshomeostasis may be efficacious in preventing age and disease-related deficits in cognition. To this end, metal chaperones (that maintain metal ion homeostasis) such as CQ and PBT2 (Prana Biotechnology) have been shown to result in the rapid recovery of cognitive performance in both APP Tg mice (Adlard et al., 2008) and normal aged wild-type mice (Adlard et al., 2014) and may also have therapeutic benefit in AD (Faux et al., 2010; Lannfelt et al., 2008; Ritchie et al., 2003). Thus, if the mechanism of action of metal chaperones was to directly modulate key plasticity-related proteins at the synapse, it would be anticipated that they would prevent the synaptic and cognitive deficits that characterise the ZnT3 KO model. Indeed, in this study, we have demonstrated that short-term CQ treatment of ZnT3 KO mice restores cognition, elevates zinc levels in the hippocampus and results in a concomitant restoration in levels of key proteins involved in learning/ memory and synaptic plasticity.
We have previously reported that ZnT3 KO mice develop an agedependent cognitive phenotype that is concomitant with the modulation of a suite of pre- and postsynaptic proteins involved in learning and memory (Adlard et al., 2010). In this study, we hypothesised that correcting these deficits would require overcoming the zincimpermeability of the blood-brain barrier. We reasoned that CQ might present zinc to postsynaptic targets, leading to events such as TrkB activation and pro-BDNF conversion, since it is a metal chaperone that enters the brain, restores cognition in amyloid protein precursor transgenic mice within days of treatment (Adlard et al., 2008) and modestly raises brain zinc in that AD model (Adlard et al., 2008; Cherny et al., 2001). Indeed, 6 weeks of CQ treatment fully restored cognitive function (both learning and recall in the Morris water maze) in the 6-month-old ZnT3 KO mice to levels equivalent to WT animals. To further profile the behavioural deficits in these mice, we conducted a Y-maze. The performance of the young ZnT3 KO mice was indistinguishable from their WT counterparts, and CQ had no effect in these animals, consistent with our observations in the water maze. In contrast, and also consistent with the water maze data, the aged ZnT3 KO mice had a significant deficit in the Y-maze that was completely prevented by CQ treatment. These data argue that zinc deficits at the glutamatergic synapse, which may occur as a function of age or disease, are sufficient to cause cognitive impairment. Furthermore, metal chaperones, that are proposed to restore metal ion homeostasis, are an effective therapeutic to prevent the cognitive decline. To address this further we examined an in vitro electrophysiological surrogate of cognition in the intact animal, LTP in hippocampal slices. These data demonstrate that the ZnT3 KO mice display an agedependent phenotype, such that young animals exhibit normal LTP while aged mice have a significant impairment compared to WT controls, consistent with the findings from the water maze and Y-maze. To determine whether passive zinc supplementation was sufficient to overcome these deficits, as compared to the use of metal chaperones, we incubated hippocampal slices with either zinc or CQ + zinc. These data demonstrate that zinc alone partially restored LTP, whereas CQ + zinc fully restored LTP in the ZnT3 KO slices. The precise mechanism underlying the CQ-mediated restoration of LTP is continuing to be explored. In whole animal studies, it has yet to be determined whether CQ is metallated when it enters the brain, but clearly these metal chaperones require the presence of zinc in order to have biological effect (Adlard et al., 2011; Crouch et al., 2011). Similarly, we also recently demonstrated that CQ and zinc alone were insufficient to rescue the phenotype present in a Zip4 intestinal KO model of acrodermatitis enteropathica, but the combination was highly effective (Geiser et al., 2013).

In the present study, it is clear, therefore, that $\mathrm{CQ}$ has a positive longterm effect on learning and memory in the ZnT3 KO model. Other reports have suggested, however, that CQ can negatively impact upon cognition. Superficially, this seems at odds with the current work, however it is consistent with the over-arching hypothesis that zinc homeostasis is critical to normal cognition. Specifically, Takeda et al. (2010, 2011) utilised either CQ or CQ + Zn injections in young (6-week-old) rats to demonstrate that the acute modulation of hippocampal zinc (resulting in either a deficit or an excess of hippocampal $\mathrm{Zn}$ respectively in their model) was sufficient to impair memory. These studies that utilised normal unimpaired young animals clearly present a different model to the one utilised in the present study. The biological benefits of $\mathrm{CQ}$ are therefore presumed to only be evidenced under conditions where there is an existing alteration in zinc homeostasis and will likely be modulated by a variety of factors such as treatment length and availability of endogenous $\mathrm{Zn}$.

The cognitive improvements observed in ZnT3 KO mice treated with CQ were concomitant with a significant restoration of key neurochemical deficits in these animals, including elevations in various pre- and postsynaptic proteins and glutamate receptors. These alterations suggest an improved brain milieu, perhaps resulting in improved neuronal health (as evidenced by elevated neurotrophic support), and perhaps 
also an increase in neuronal number (as evidenced by elevated synaptophysin (surrogate marker for synapses), spinophilin (surrogate marker for dendritic spines) and doublecortin (surrogate marker for immature neurons)). These changes could cumulatively contribute to the increased performance on various behavioural tasks. However, one standout neurochemical difference was a rebounding elevation in NMDAR2b protein levels in the CQ-treated mice. This is a phenomenon that was also observed in PBT2-treated aged wild-type mice (Adlard et al., 2014), where similar improvements in learning and memory were also observed. The genetic over-expression of NMDAR2b has previously been shown to result in enhanced learning and memory performance in various rodent behavioural tasks (Tang et al., 1999), suggesting that a primary mediator of the cognitive benefit derived from metal chaperone treatment may be related to a direct effect on synaptic glutamate receptors, particularly NMDAR2b. Given how closely these data align to our recent publication with PBT2 (Adlard et al., 2014), and also given the known metal chaperone effect of CQ (Adlard et al., 2008), we examined brain zinc content as a potential upstream mediator of the neurochemical alterations that may underlie the improved learning and memory. Consistent with our hypothesis that 8-hydroxy quinolones such as CQ function to restore metal ion homeostasis, we observed a small but significant increase in hippocampal zinc levels following CQ treatment. While these data are convincing, there are several important caveats around their interpretation. First, the ICPMS analysis is performed in bulk extracts of the hippocampus-so there is no regional specificity to the zinc increase observed using this methodology. In contrast, with the synchrotron analysis where we were able to achieve spatial localisation of zinc levels, we encountered technical difficulties that meant we could only image the dentate (this region, particularly the polymorph layer/hilus, is a zinc-rich area where a variety of cell types are found, including glutamatergic mossy cells that have significant dendritic arborisation; granule cell axons innervate and synapse within this area as well, and mossy fiber axons also have a set of collaterals that innervate this part of the brain). Clearly, the LTP measurements and also the behavioural outputs are more dependent upon other areas of the hippocampus (such as CA1). As we cannot assume that the increase in zinc levels in the dentate will translate to a parallel increase in zinc in other regions of the hippocampus, then we cannot definitively draw the conclusion that there was a CQ-induced increase in zinc in an appropriate region of the brain that mediated the biochemical and behavioural effects we observed. Furthermore, it is not clear if the elevated zinc reflects "free" ionic zinc that is able to bind and modulate receptor activity, or if it is instead sequestered by various transporters (such as metallothioneins, which, for example, have a much greater affinity for zinc than CQ). Thus, more detailed imaging studies examining free ionic zinc, together with the localisation of the increased zinc would strengthen our conclusions. In this latter regard, it would be instructive to know whether $\mathrm{Zn}$ is still being packaged into synaptic vesicles in the absence of ZnT3. Could CQ be acting as a surrogate zinc transporter? One possibility is that other transporters such as vGlut1 may compensate for the lack of ZnT3 to facilitate zinc-loading of the synaptic vesicles. We recently postulated this as a potential mechanism of synaptic zinc increase following metal chaperone treatment (Adlard et al., 2014). These studies are an ongoing direction of our lab.

However, while it is important to acknowledge these caveats, these data are consistent with our previous reports on the metal chaperone activity of this class of compound, and also with the known role of zinc in learning and memory, where among other things it functions as a neuronal messenger and a modulator of synaptic transmission (Paoletti et al., 2009; Smart et al., 2004). The weight of evidence, therefore, lies in favour of CQ mediating its apparent benefits via a modulation of hippocampal zinc levels within this model. Given the apparent small magnitude of this change, however, one intriguing avenue of investigation moving forward is to try and understand whether there is a minimum "zinc threshold" that may predict the biochemical and behavioural effects observed in this, and previous, studies.
Taken together these data demonstrate that under conditions of cellular metal deficiency within the brain, CQ facilitates a partial restoration of zinc homeostasis and also restores key zinc-dependent proteins and signalling pathways that cumulatively effect cognitive performance. These data support the use of metal chaperones for the prevention of metal-dependent cognitive decline, which may occur as a function of both age and disease.

\section{Conflict of Interest}

PAA, DIF and AIB are shareholders in Prana Biotechnology Ltd. PAA and DIF are also consultants to Prana Biotechnology Ltd. AIB is also a shareholder in Mesoblast Ltd, Cogstate Ltd, Brighton LLC, Eucalyptus LLC, and is a paid consultant for Collaborative Medicinal Discovery LLC and Brighton LLC.

\section{Acknowledgements}

We thank Milawaty Nurjono and Gulay Filiz for assistance with experiments. PAA, DIF and AIB are supported by the National Health and Medical Research Council. PAA is also supported by the Australian Research Council. The Florey Institute of Neuroscience and Mental Health acknowledges the strong support from the Victorian Government and in particular the funding from the Operational Infrastructure Support Grant. Parts of this research were undertaken on the X-ray Fluorescence Microscopy beamline at the Australian Synchrotron, Victoria, Australia.

\section{References}

Adlard, P.A., Cherny, R.A., Finkelstein, D.I., Gautier, E., Robb, E., et al., 2008. Rapid restoration of cognition in Alzheimer's transgenic mice with 8-hydroxy quinoline analogs is associated with decreased interstitial Abeta. Neuron 59, 43-55.

Adlard, P.A., Parncutt, J.M., Finkelstein, D.I., Bush, A.I., 2010. Cognitive loss in zinc transporter-3 knock-out mice: a phenocopy for the synaptic and memory deficits of Alzheimer's disease? J. Neurosci. Off. J. Soc. Neurosci. 30, 1631-1636.

Adlard, P.A., Bica, L., White, A.R., Nurjono, M., Filiz, G., et al., 2011. Metal ionophore treatment restores dendritic spine density and synaptic protein levels in a mouse model of Alzheimer's disease. PLoS One 6, e17669.

Adlard, P.A., Sedjahtera, A., Gunawan, L., Bray, L., Hare, D., et al., 2014. A novel approach to rapidly prevent age-related cognitive decline. Aging Cell 13, 351-359.

Besser, L., Chorin, E., Sekler, I., Silverman, W.F., Atkin, S., et al., 2009. Synaptically released zinc triggers metabotropic signaling via a zinc-sensing receptor in the hippocampus. J. Neurosci. Off. J. Soc. Neurosci. 29, 2890-2901.

Cherny, R.A., Atwood, C.S., Xilinas, M.E., Gray, D.N., Jones, W.D., et al., 2001. Treatment with a copper-zinc chelator markedly and rapidly inhibits beta-amyloid accumulation in Alzheimer's disease transgenic mice. Neuron 30, 665-676.

Crouch, P.J., Savva, M.S., Hung, L.W., Donnelly, P.S., Mot, A.I., et al., 2011. The Alzheimer's therapeutic PBT2 promotes amyloid-beta degradation and GSK3 phosphorylation via a metal chaperone activity. J. Neurochem. 119, 220-230.

Faux, N.G., Ritchie, C.W., Gunn, A., Rembach, A., Tsatsanis, A., et al., 2010. PBT2 rapidly improves cognition in Alzheimer's disease: additional phase II analyses. J. Alzheimers Dis. 20, 509-516.

Frederickson, C.J., Koh, J.Y., Bush, A.I., 2005. The neurobiology of zinc in health and disease. Nat. Rev. Neurosci. 6, 449-462.

Frederickson, C.J., Giblin III, L.J., Balaji, R.V., Masalha, R., Zeng, Y., et al., 2006. Synaptic release of zinc from brain slices: factors governing release, imaging, and accurate calculation of concentration. J. Neurosci. Methods 154, 19-29.

Geiser, J., De Lisle, R.C., Finkelstein, D., Adlard, P.A., Bush, A.I., et al., 2013. Clioquinol synergistically augments rescue by zinc supplementation in a mouse model of acrodermatitis enteropathica. PLoS One 8, e72543.

Huang, Y.Z., Pan, E., Xiong, Z.Q., McNamara, J.O., 2008. Zinc-mediated transactivation of TrkB potentiates the hippocampal mossy fiber-CA3 pyramid synapse. Neuron 57 546-558.

Lannfelt, L., Blennow, K., Zetterberg, H., Batsman, S., Ames, D., et al., 2008. Safety, efficacy, and biomarker findings of PBT2 in targeting Abeta as a modifying therapy for Alzheimer's disease: a phase Ila, double-blind, randomised, placebo-controlled trial. Lancet Neurol. 7, 779-786.

Lee, J.Y., Cole, T.B., Palmiter, R.D., Suh, S.W., Koh, J.Y., 2002. Contribution by synaptic zinc to the gender-disparate plaque formation in human Swedish mutant APP transgenic mice. Proc. Natl. Acad. Sci. U. S. A. 99, 7705-7710

Lee, J.Y., Kim, Y.J., Kim, T.Y., Koh, J.Y., Kim, Y.H., 2008. Essential role for zinc-triggered p75NTR activation in preconditioning neuroprotection. J. Neurosci. Off. J. Soc. Neurosci. 28, 10919-10927.

Lei, P., Ayton, S., Finkelstein, D.I., Spoerri, L., Ciccotosto, G.D., et al., 2012. Tau deficiency induces parkinsonism with dementia by impairing APP-mediated iron export. Nat Med. 18, 291-295. 
Paoletti, P., Vergnano, A.M., Barbour, B., Casado, M., 2009. Zinc at glutamatergic synapses. Neuroscience 158, 126-136.

Ritchie, C.W., Bush, A.I., Mackinnon, A., Macfarlane, S., Mastwyk, M., et al., 2003. Metalprotein attenuation with iodochlorhydroxyquin (clioquinol) targeting Abeta amyloid deposition and toxicity in Alzheimer disease: a pilot phase 2 clinical trial. Arch. Neurol. 60, 1685-1691.

Smart, T.G., Hosie, A.M., Miller, P.S., 2004. Zn2 + ions: modulators of excitatory and inhibitory synaptic activity. Neuroscientist 10, 432-442.
Takeda, A., Takada, S., Ando, M., Itagaki, K., Tamano, H., et al., 2010. Impairment of recognition memory and hippocampal long-term potentiation after acute exposure to clioquinol. Neuroscience $171,443-450$.

Takeda, A., Takada, S., Nakamura, M., Suzuki, M., Tamano, H., et al., 2011. Transient increase in $\mathrm{Zn} 2+$ in hippocampal CA1 pyramidal neurons causes reversible memory deficit. PLoS One 6, e28615.

Tang, Y.P., Shimizu, E., Dube, G.R., Rampon, C., Kerchner, G.A., et al., 1999. Genetic enhancement of learning and memory in mice. Nature 401, 63-69. 